\title{
Fingerprint Profile of Selected Ayurvedic Churnas/Preparations:
}

\section{An Overview}

Garg S, Mishra A and Gupta R*

Faculty of Pharmacy, Babu Banarasi Das National Institute of Technology and Management (BBD University), Lucknow, 227105 (U.P), India

\begin{abstract}
Ayurvedic formulations [such as solid dosage (vati, churna), semisolid (avaleha, ghritas), liquid (asava, arishta)] have numerous uses in Ayurveda. They effect or help to rectify the three doshas in the body, and restore homeostatic balance that builds up in the body's digestive system and spreads to the tissues. Standardization and analysis of the chemical marker of the Ayurvedic and other poly herbal formulation has always been a concern. For Researchers, Standardization is the need of hour for the present era to set standards for maintaining the quality and efficacy of the herbal products. HPTLC offers major advantages over other conventional chromatographic techniques such as unsurpassed flexibility (esp. stationary and mobile phase), choice of detection wavelength, user friendly, rapid and cost effective/economical. The present study compiles the progress made on the development of optimized and validated HPTLC/HPLC method for the simultaneous estimation of markers in different ayurvedic churnas/ preparations. HPTLC/HPLC profile is quite helpful in setting up of standards. The present study is an attempt to compile the major studies carried out on Ayurvedic preparations like: Churnas, avaleha, asava, arishta, vati, rasa, taila, ghritas and herbal capsules etc. which may be of use to develop/compile the fingerprint profile for evaluating the purity \& quality of ayurvedic formulations, thus helpful as a reference in developing pharmacopoeial standards (Table 3).
\end{abstract}

Keywords: Ayurvedic formulations; Churnas; Standardization; Markers; Fingerprint; HPTLC

\section{Introduction}

Ayurvedic formulations have numerous uses in Ayurveda. They effect or help to rectify the three doshas or humors in the body [1]. Churna is a fine powder of well dried drug or drugs described in ancient literature [2]. Quantitative estimation of chemical markers of each ingredient in the poly herbal preparation required ideal separation technique [3,4]. For herbal preparations (including polyherbal), there is an urgent need for scientific proof/validation with chemical standardization protocols/procedures, biological assays, animal models and clinical trials $[3,5]$. HPTLC thus offers major advantages over other commonly available conventional chromatographic techniques [4]. The proposed method was validated on the basis of its selectivity, linearity, limit of detection (LOD) and limit of quantification (LOQ) according to ICH requirements $[3,6]$. HPTLC profile is quite helpful in setting up of standards for evaluating the purity and quality of Ayurvedic preparations. This will be helpful to overcome batch to batch variations in different Ayurvedic churna/preparations [7]

\section{Churna}

Churna is a fine powder of a drug or drugs which is prepared by mixing clean, finely powdered and sieved drugs. The term churna may be applied to the powder prepared by a single drug or a combination of more drugs [8]. Ayurvedic formulary of India has given the specification for the composition of churnas $[9,10]$

Chaturjat churna: A polyherbal formulation consisting of 4 ingredients with specific morphological parts. The ingredients are Cinnamomum zeylanicum, Elettaria cardamomum, Cinnamomum tamala and Tribulus terrestris [11].

HPTLC profile: The crude drug sample extracted in Methanol (150 $\mathrm{ml} \times 5$ ) through Soxhlet apparatus was filtered and concentrated to 5-10 ml. High Performance Thin Layer Chromatography was carried out by applying $6 \mu$ of the sample on TLC Silica gel plate 60 F 254 (from
Merck India Ltd, Germany) and developed the plate to a distance of 10 cm using Toluene: Ethyl acetate (9:1) as mobile phase, examined under Ultra Violet Light at $254 \mathrm{~nm}$; and under $366 \mathrm{~nm}$; after derivatization with $5 \%$ methanolic sulphuric acid solution different $\mathrm{R}_{f}$ value in TLC finger print was found to be $0.24,0.47,0.54,0.76,0.80,0.84$, and 0.92 . HPTLC finger printing profile of Caturjata churna was also developed in Toluene: Ethyl acetate (93:7) solvent system (Table 1) [12] (Figure 1).

Pancasama churna: Pancasama churna, a polyherbal formulation consists of rhizomes of Cyprus rotandus (Mustha), whole plant Termenalia chebula (haritaki), fruit of Piper longum (pippali), root of Operculina turpethum (Trivrat) and sandha lavana [7].

TLC/HPTLC Analysis: TLC and HPTLC finger printing profile of Pancasama Churna (ethanol extract) were developed in Toluene: Ethyl acetate: Formic acid (5.0:3.5:1.0 v/v) solvent system (Figure 2).

Triphala churna: It is an age old commonly used Ayurvedic powdered preparation in Indian systems of medicine. Ayurvedic formulary of India has given the specification for the composition of Triphla churna $[9,10]$. This well known formulation is made by combining Terminalia chebula, Terminalia belerica and Embellica officinalis, in equal proportions [13].

HPTLC: A HPTLC-densitometric method of analysis for markers

*Corresponding author: Rajiv Gupta, Pharmacy, Babu Banarasi Das Nationa Institute of Technology \& Management (BBD University), BBD Green City, Lucknow-227105, (U.P.), India, Tel: +91 9839278227, 05223911132; E-mail: rajiv961@rediffmail.com

Received June 05, 2013; Accepted June 25, 2013; Published June 28, 2013

Citation: Garg S, Mishra A, Gupta R (2013) Fingerprint Profile of Selected Ayurvedic Churnas/Preparations:An Overview. Altern Integ Med 2: 125. doi:10.4172/2327 5162.1000125

Copyright: (c) 2013 Garg S, et al. This is an open-access article distributed unde the terms of the Creative Commons Attribution License, which permits unrestricted use, distribution, and reproduction in any medium, provided the original author and source are credited. 


\begin{tabular}{|c|c|c|c|c|}
\hline S.No. & Churna/extract & Constituents & $\begin{array}{l}\text { Solvent system and Scanning } \\
\text { wavelength }\end{array}$ & Standard \\
\hline 1. & $\begin{array}{l}\text { Ajmodadi churna (Methanol) } \\
\text { [3] }\end{array}$ & $\begin{array}{l}\text { Piper species (Piper longum in both form root and fruit and Piper } \\
\text { nigrum) [10] }\end{array}$ & Toluene: Ethyl acetate $(07: 03)$ at $336 \mathrm{~nm}$. & Piperine \\
\hline 2. & $\begin{array}{l}\text { Amukkara choornam } \\
\text { (toluene) [30] }\end{array}$ & $\begin{array}{l}\text { Piper nigrum, Piper longum, Zingiber officinale, Amukkara } \\
\text { (Withania somnifera), Elletaria cardamomum, Cinnamomum } \\
\text { wightii, Syzygium aromaticum }\end{array}$ & $\begin{array}{l}\text { Toluene: Ethyl acetate }(9: 3 \mathrm{v} / \mathrm{v}) \text { at } 260 \\
\mathrm{~nm} .\end{array}$ & Not mentioned \\
\hline 3. & $\begin{array}{l}\text { Chaturjat churna (Methanol) } \\
\text { [11] }\end{array}$ & $\begin{array}{l}\text { Cinnamomum zeylanicum, Elettaria cardamomum, Cinnamomum } \\
\text { tamala and Tribulus terrestris }\end{array}$ & Toluene: Ethyl acetate $(9: 1)$ at $254 \mathrm{~nm}$. & Not mentioned \\
\hline 4. & $\begin{array}{l}\text { Hingashtak Churna } \\
\text { (methanol) [31] }\end{array}$ & Piper longum, Piper nigrum, Curcuma longa, Thymus vulgaris & $\begin{array}{l}\text { Toluene-ethyl acetate-methanol, 9:1:0.5 } \\
\text { at } 420,333 \text {, and } 277 \mathrm{~nm}\end{array}$ & $\begin{array}{l}\text { Curcumin, piperine, } \\
\text { and thymol }\end{array}$ \\
\hline 5. & $\begin{array}{l}\text { Kuberaksha/Kantaki karanja } \\
\text { patra Churna (methanol) } \\
{[32]}\end{array}$ & Caesalpinia bonduc & $\begin{array}{l}\text { Ethyl acetate: methanol: water } \\
(100: 13.5: 10) \text { at } 254 \mathrm{~nm} .\end{array}$ & Not mentioned \\
\hline 6. & $\begin{array}{l}\text { Laghugangadhar Churna } \\
\text { (methanol) [1] }\end{array}$ & $\begin{array}{l}\text { Cyperus rotundus, Symplocos racemosa, Woodfordia fruticosa, } \\
\text { Aegle marmelos, }\end{array}$ & $\begin{array}{l}\text { Toluene: Ethyl acetate }(90: 10) \text { at } 254 \text { and } \\
366 \mathrm{~nm} \text {. }\end{array}$ & Not mentioned \\
\hline 7. & $\begin{array}{l}\text { Nisamalaki churna } \\
\text { (Methanol and Aqueous) } \\
{[33]}\end{array}$ & $\begin{array}{l}\text { Curcuma longa; Emblica } \\
\text { Officinalis }\end{array}$ & $\begin{array}{l}\text { Chloroform-methanol }(9.5: 0.5 \mathrm{v} / \mathrm{v}) \text {, Ethanol- } \\
\text { glacial acetic acid }(9: 1 \mathrm{v} / \mathrm{v}) \text { at } 500 \mathrm{~nm}\end{array}$ & $\begin{array}{l}\text { Curcumin; } \\
\text { Ascorbic acid }\end{array}$ \\
\hline 8. & $\begin{array}{l}\text { Pancasma churna(Ethanol) } \\
\text { [7] }\end{array}$ & $\begin{array}{l}\text { Operculina turpethum; Terminalia chebula, Cyprus rotundus; } \\
\text { Piper longum }\end{array}$ & $\begin{array}{l}\text { Toluene: ethyl acetate: Formic acid } \\
(5.0: 3.5: 1.0 \mathrm{v} / \mathrm{v}) \text { at } 366 \mathrm{~nm} \text {. }\end{array}$ & $\begin{array}{l}\text { Piperine and gallic } \\
\text { acid }\end{array}$ \\
\hline 9. & $\begin{array}{l}\text { Panchaskar churna } \\
\text { (methanol) }[34,35]\end{array}$ & $\begin{array}{l}\text { Cassia angustifolia, Foeniculum vulgare, } \\
\text { Terminalia chebula, Zingiber officinale, Anethum sowa, Rock salt } \\
\text { (Saindhava lavana). }\end{array}$ & Toluene: ethyl acetate at $260 \mathrm{~nm}$ & Not mentioned \\
\hline 10. & $\begin{array}{l}\text { Palas abijadi churna } \\
\text { (methanol) [36] }\end{array}$ & $\begin{array}{l}\text { Butea monosperma; Holarrhena antidysentrica,embelia ribes, } \\
\text { Azadirachta indica, Swertia chirata }\end{array}$ & $\begin{array}{l}\text { Toluene: Ethyl acetate }(90: 10 \mathrm{v} / \mathrm{v}) \text { at } 260 \\
\mathrm{~nm} .\end{array}$ & Not mentioned \\
\hline 11. & Panchkol Churna [37] & $\begin{array}{l}\text { Piper longum, Piper nigrum, Cuminum cyminum, Plumbago } \\
\text { zeylanica, Embelia ribes, Zingiber officinale }\end{array}$ & $\begin{array}{l}\text { Toluene: ethyl acetate }(7: 3) \text { at } 340,420 \\
\mathrm{~nm} \text {. }\end{array}$ & $\begin{array}{l}\text { Piperine, plumbagine, } \\
\text { zingiberine }\end{array}$ \\
\hline 12. & $\begin{array}{l}\text { Triphla Churna (aqueous) } \\
{[13,14]}\end{array}$ & Terminalia chebula, Terminalia belerica and Embellica officinalis & $\begin{array}{l}\text { Ethanol: glacial acetic acid: toluene } \\
(5.5: 1: 1.5) \text { for ascorbic acid and Ethyl } \\
\text { acetate: toluene: acetone }(4.5: 4: 1) \text { for } \\
\text { gallic acid at } 254 \mathrm{~nm} \text {. }\end{array}$ & $\begin{array}{l}\text { Ascorbic acid and } \\
\text { gallic acid [13] }\end{array}$ \\
\hline 13. & $\begin{array}{l}\text { Trikatu Churna (methanol) } \\
\text { [15] }\end{array}$ & Piper longum, Piper nigrum, Zingiber officinale & $\begin{array}{l}\text { Toulene: ethylacetate: glacial acetic acid } \\
(8: 2: 0.1 \mathrm{v} / \mathrm{v} / \mathrm{v}) \text { at } 550 \mathrm{~nm} \text {. }\end{array}$ & Piperine \\
\hline 14. & $\begin{array}{l}\text { Talishadi churna (methanol) } \\
\text { [38] }\end{array}$ & $\begin{array}{l}\text { Piper longum, Piper nigrum, Zingiber officinale, Elletaria } \\
\text { cardamomum, Cinnamomum zeylanicum, Bambusa arundinacea, }\end{array}$ & $\begin{array}{l}\text { Toluene: ethyl acetate }(9: 3 \mathrm{v} / \mathrm{v}) \text { at } 260 \\
\mathrm{~nm} \text {. }\end{array}$ & Not mentioned \\
\hline 15. & $\begin{array}{l}\text { Vidanga churna (methanol) } \\
\text { [39] }\end{array}$ & Embelia ribes & $\begin{array}{l}\text { Chloroform: ethyl acetate: formic acid } \\
(5: 4: 1 \mathrm{v} / \mathrm{v} / \mathrm{v}) \text { at } 291 \mathrm{~nm} \text {. }\end{array}$ & Embelin \\
\hline 16. & $\begin{array}{l}\text { Ashwagandha churna } \\
\text { (methanol) }[40,41]\end{array}$ & Withania somnifera & $\begin{array}{l}\text { Toluene: ethyl acetate: formic acid at } \\
540 \mathrm{~nm} \text {. }\end{array}$ & $\begin{array}{l}\text { Beta-sitosterol D } \\
\text { glucoside }\end{array}$ \\
\hline
\end{tabular}

Table 1: HPTLC finger print of different churnas.

i.e. Gallic acid [14] and ascorbic acid in Triphla churna (methanol extract) was developed. Water was selected as a solvent for preparing standard solutions.

Quantitative estimation of gallic acid and ascorbic acid was performed separately on aluminum backed silica gel 60 F254 TLC plates $(10 \mathrm{~cm} \times 10 \mathrm{~cm}$ plate size, layer thickness $0.2 \mathrm{~mm}$, E-Merck, Darmstadt, Germany). Ascorbic acid shows $\mathrm{R}_{\mathrm{f}}$ value of $0.74 \pm 0.1$ using ethanol: glacial acetic acid: toluene (5.5:1:1.5) and gallic acid showed $R_{f}$ value of $0.54 \pm 0.1$, using ethyl acetate : toluene : acetone (4.5:4:1) as mobile phase, scanned at $254 \mathrm{~nm}$. Thus a simple, precise and accurate method for quantitative estimation of ascorbic acid and gallic acid in herbal medicine (triphala churna) by HPTLC was developed. The gallic acid and ascorbic acid content in triphala churna was quantified (Figure 3).

Trikatu churna: Trikatu Churna is well known Ayurvedic Formulation, comprised of the fruits of two medicinal important plants of Piper longum (Pipali) along with Piper nigrum (Marica) and rhizomes of Zingiber officinalis (Saunth) [15].

HPTLC: The fingerprint method for Trikatu churna by simple high performance thin layer chromatography (HPTLC) determination using piperine as a standard, which is as an important and major content in formulation. The concentration of piperine present in raw materials was found to be $4.2 \% \pm 0.43 \mathrm{w} / \mathrm{w}$ in Piper nigrum (Maricha), and $2.15 \%$ $\pm 0.68 \mathrm{w} / \mathrm{w}$ in Piper longum (Pipali) respectively and in three identical laboratory batch of Trikatu churna name TK-I, TK-II, TK-III, was $2.13 \% \pm 0.62,2.42 \% \pm 0.67,2.18 \% \pm 0.41 \mathrm{w} / \mathrm{w}$ respectively with mean value $2.24 \% \pm 0.48 \mathrm{w} / \mathrm{w}$. The piperine content of all the three batches is found to be in close proximities with each other. Obtained results were compared with marketed formulations. Better results were obtained with mobile phase consisting of Toulene: ethylacetate: glacial acetic acid $(8: 2: 0.1 \mathrm{v} / \mathrm{v} / \mathrm{v})$ at $550 \mathrm{~nm}$ gave Rf values of $0.42 \pm 0.03$ for piperine at $550 \mathrm{~nm}$.

Better results were also obtained with mobile phase consisting of toluene: ethyl acetate $(70: 30 \mathrm{v} / \mathrm{v})$, gave $\mathrm{Rf}$ values of $0.42 \pm 0.03$ for piperine [16] (Figure 4).

Haritaki churna: Haritaki churna mainly constitutes of dried fruit of Terminalia chebula [17].

HPLC: A high performance liquid chromatography method coupled with diode array detection was developed to simultaneously determine seven different marker compounds in Haritaki churna, an ayurvedic formulation. These markers are gallic acid, methyl gallate, ethyl gallate, ellagic acid, chebulagic acid, chebulinic acid and pentaO-galloyl- $\beta$-D-glucose. HPLC analysis was carried out at wavelength $272 \mathrm{~nm}$. The chromatographic separation was performed on Thermo 

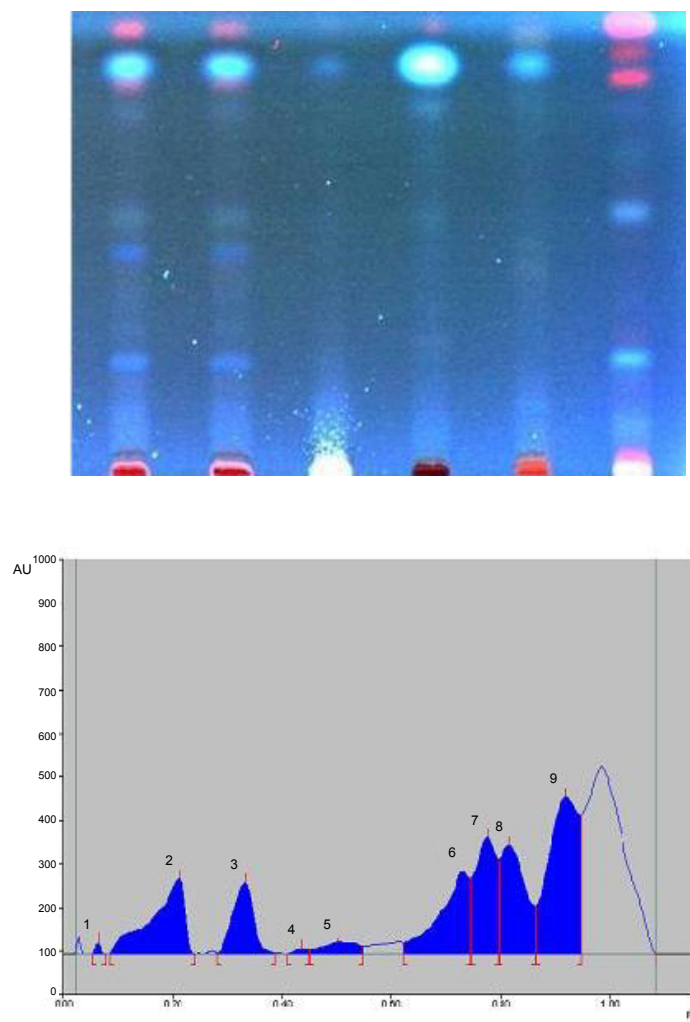

Figure 1: TLC Finger prints of test solution of Chaturjata Churna at $366 \mathrm{~nm}$ \& HPTLC fingerprint of Caturjata churna at $254 \mathrm{~nm}[12]$.

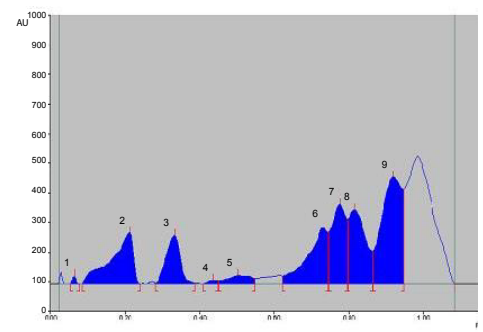

$254 \mathrm{~nm}$ $366 \mathrm{~nm}$

After Derivatisation
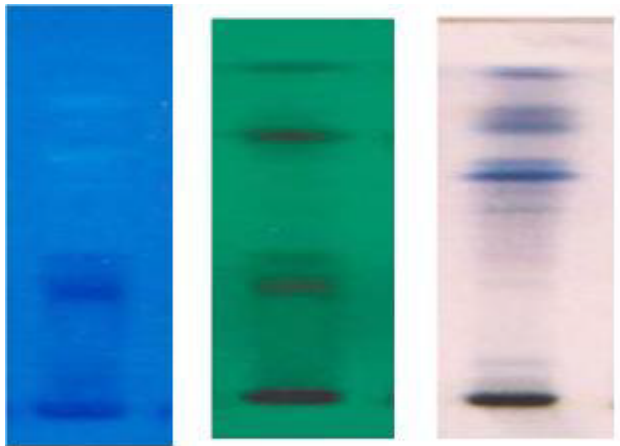

Figure 2: HPTLC Finger print of Pancasama Churna at $254 \mathrm{~nm}$ and Thin layer chromatography of Pancasama Churna (Toluene: Ethyl acetate: Formic acid :5.0:3.5:1.0 v/v) [7].
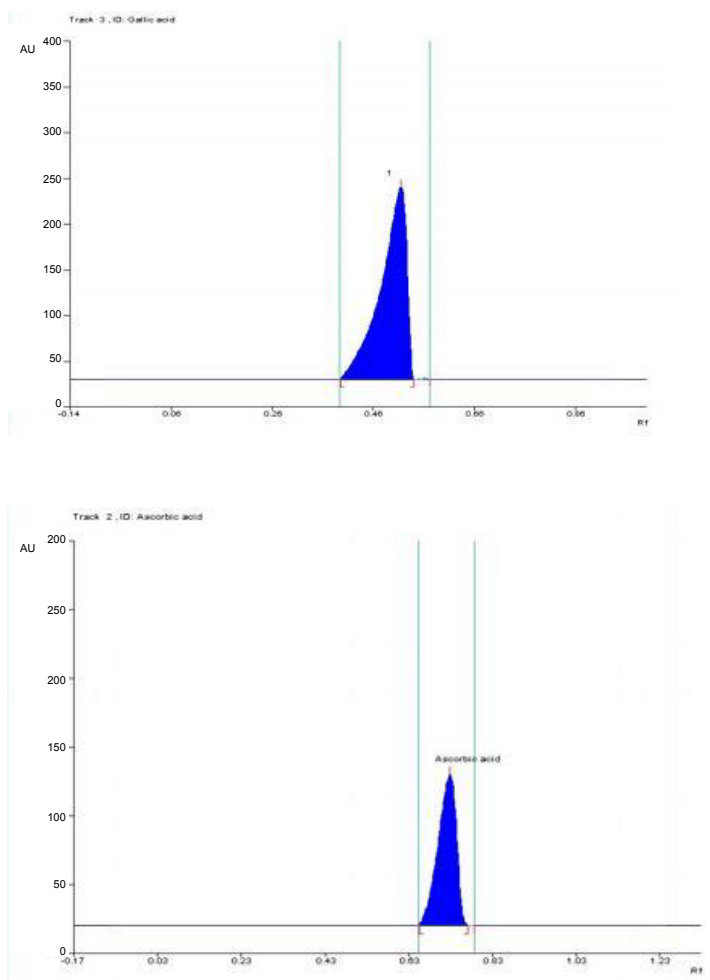

Figure 3: Densitogram of gallic acid spot was measured at $254 \mathrm{~nm}$ using mobile phase, ethyl acetate: toluene: acetone (4.5:4:1) and Densitogram of ascorbic acid was measured at $254 \mathrm{~nm}$ using mobile phase ethanol: glacial acetic acid: toluene $(5.5: 1: 1.5)$ [13].

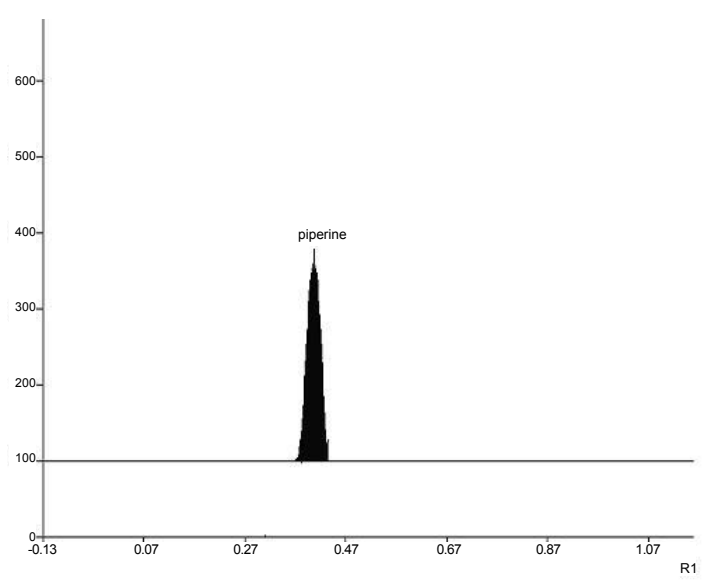

Figure 4: HPTLC chromatogram of Piperine $\left(R_{f}=0.42\right)$ [16].

Scientific BDS HYPERSIL Phenyl reversed-phase column $(100 \mathrm{~mm} \times 4.6$ $\mathrm{mm}, 3 \mu \mathrm{m})$. The mobile phase was consisted of $0.02 \%$ triethyl amine aqueous $\mathrm{pH} 3.0$ with ortho-phosphoric acid (A) and acetonitrile (B) at a flow rate of $1.0 \mathrm{ml} / \mathrm{min}$ gradient mode. The flow rate was $1.0 \mathrm{ml} /$ min and aliquots of $10 \mu \mathrm{l}$ were injected. Regression equations showed good linear relationships (R2>0.998) between the peak area of each marker and concentration. In this study, an HPLC-DAD method for the qualification and quantification of phtyoconstituents in Haritaki churna has been developed and successfully applied for comparison of three marketed samples (HC1, HC2, and HC3) (Table 2). 


\begin{tabular}{|c|c|c|c|c|}
\hline S. No. & Churna/extract & Constituents & Solvent system and Scanning wavelength & Standard \\
\hline 1. & $\begin{array}{l}\text { Haritaki churna (methanol: } \\
\text { water } 70: 30)[17]\end{array}$ & Terminalia chebula & $\begin{array}{l}\text { Tri ethyl amine aq. } \mathrm{pH}: 3 \text { with Orthophosphoric acid } \\
\text { and acetonitrile at } 272 \mathrm{~nm} \text { and flow rate of } 1 \mathrm{ml} / \mathrm{min} \text {, } \\
\text { an injection volume of } 10 \mu \mathrm{l} .\end{array}$ & $\begin{array}{l}\text { Gallic acid, ellagic } \\
\text { acid }\end{array}$ \\
\hline 2. & $\begin{array}{l}\text { Lavanbhaskar churna } \\
\text { (ethanol) [42] }\end{array}$ & $\begin{array}{l}\text { Piper longum, Piper nigrum, Zingiber offcinalis, Elleteria } \\
\text { cardamomum, Cinnamomum zeylanicum, Punica } \\
\text { gratum seeds, Cuminum cymenum, Salts (sea salt, } \\
\text { black salt, rock salt and vida salt) }\end{array}$ & $\begin{array}{l}\text { Methanol: water }(69: 31) \text { at } 343 \mathrm{~nm} \text { and injection } \\
\text { volume: } 20 \mu \mathrm{l} \text { and flow rate were } 1.5 \mathrm{ml} / \mathrm{min}\end{array}$ & Piperine \\
\hline 3. & $\begin{array}{l}\text { Sitopaladi Churna (ethanol) } \\
\text { [43] }\end{array}$ & $\begin{array}{l}\text { Piper nigrum, Piper longum, Zingiber officinale, Elletaria } \\
\text { cardamomum, Cinnamomum wightii, sugar, Bombusa } \\
\text { bombos. }\end{array}$ & $\begin{array}{l}\text { Methanol at } 342 \mathrm{~nm} \text { and flow rate of } 1.2 \mathrm{ml} / \mathrm{min} \text {, an } \\
\text { injection volume of } 20 \mu \mathrm{g} \text {. }\end{array}$ & Piperine \\
\hline 4. & $\begin{array}{l}\text { Trikatu Churna (methanol) } \\
\text { [44] }\end{array}$ & Piper longum, Piper nigrum, Zingiber officinale & $\begin{array}{l}\text { Methanol at } 342 \mathrm{~nm} \text { and flow rate of } 1.2 \mathrm{ml} / \mathrm{min} \text {, an } \\
\text { injection volume of } 20 \mu \mathrm{g} \text {. }\end{array}$ & Piperine \\
\hline 5. & $\begin{array}{l}\text { Triphla churna (Hexane and } \\
\text { acetonitrile) [45] }\end{array}$ & $\begin{array}{l}\text { Terminalia chebula, Terminalia belerica and Embellica } \\
\text { officinalis }\end{array}$ & $\begin{array}{l}\text { Acetonitrile: acetic acid } 99.9: 0.10 \text { at } 212 \mathrm{~nm} \text {. Flow } \\
\text { rate } 1 \\
\mathrm{ml} / \mathrm{min} \text {, Injection Volume } 10 \mu \mathrm{L}\end{array}$ & Endosulfan \\
\hline 6. & $\begin{array}{l}\text { Vidanga churna (Cholroform } \\
\text { extract sonicated with } \\
\text { methanol) [46] }\end{array}$ & Embelia ribes & $\begin{array}{l}\text { Methanol: phosphate buffer } \mathrm{pH} 3(90: 10) \text { at } 291 \mathrm{~nm} \\
\text { and flow rate of } 1.4 \mathrm{ml} / \mathrm{min} \text {, an injection volume of } \\
20 \mu \mathrm{l} \text {. }\end{array}$ & Embelin \\
\hline
\end{tabular}

Table 2: HPLC fingerprint of churnas.
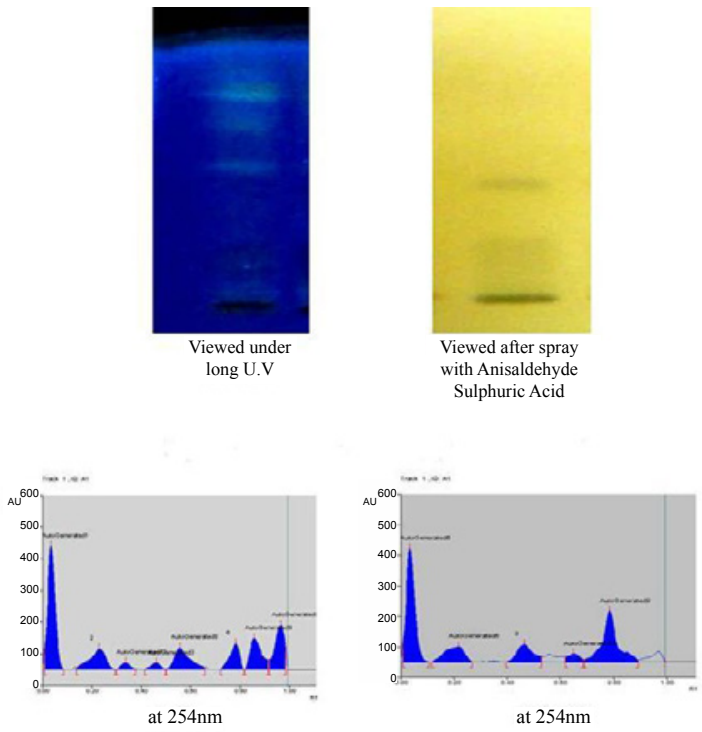

at $254 \mathrm{~nm}$

Figure 5: HPTLC Study on Vyaghrihareetaki avaleha [18]

This method is validated for good accuracy, repeatability and precision, and can be used to evaluate the quality of the drug. This multi-phytoconstituents assay method will be helpful to quality control and stability studies of Haritaki churna.

\section{Avaleha}

It is a semi-solid preparation of the drugs, prepared with addition of jaggery, sugar or sugar-candy and boiled with prescribed juices or decoctions. It acquires the consistency of a thick paste. The other similar forms are known as Modaka, Guda, Khanda, Lehya, Praasa etc. E.g: Vasa Avaleha, Chyavanprasha Avaleha, Kushmanda Avaleha etc. [8].

Vyaghrihareetaki avaleha: An Ayurvedic formulation, Vyaghrihareetaki avaleha (VHA) is a potent drug indicated for shwasa (Asthma), kasa (cough) etc. and used in the management of Tamaka Shwasa (Bronchial Asthma). Its consists of Solanum xanthocarpum, Terminalia chebula, Piper nigrum, Piper longum, Zingiber officinale, Cinnamomum zeylanicum, Cinnamomum tamala Elettaria cardamomum, Mesua ferrea, honey, jaggery [18].

High Performance Thin layer chromatography (HPTLC): Eight spots were observed in short wave UV $254 \mathrm{~nm}$ and five spots in long wave uv $366 \mathrm{~nm}$. HPTLC fingerprinting for Vyaghrihareetaki Avaleha (methanol extract) with solvent system Toluene: Ethyl acetate: Glacial acetic acid: Formic acid (5:5:1:0.5) at 254 and $336 \mathrm{~nm}$., reveals eight spots of rf values $0.01,0.14,0.30,0.41,0.50,0.72,0.82,0.92$ in short wave UV $254 \mathrm{~nm}$. In long wave UV $366 \mathrm{~nm}$ five spots at $0.01,0.12,0.40$, 0.62, 0.69 Rf values were observed (Figure 5).

Ikshvadi avaleha: Ikshvadi Avaleha is very safe to be used for tuberculosis in children. Its consists of Phyllanthus urinaria, Saccharum officinarum, Bambusa arundinacea, Mucuna prurita, Piper nigrum, Cinnamomum zeylanicum, Elettaria cardamomum, and honey [19].

HPTLC: TLC and HPTLC were carried out after organizing appropriate solvent system in which maximum 4 spots were distinguished in TLC and 3 spots in HPTLC and most of the Rf values were identical when done with different sample extractive methods. It is inferred that the formulation meets the minimum qualitative standards as reported in the API at a preliminary level.

HPTLC study of the Unsaponifiable fraction of the Ikshvadi Avaleha (methanol extract) was also carried out by using the same solvent system of Toluene: Ethyl acetate $(7: 3 \mathrm{v} / \mathrm{v})$. After completion of HPTLC post chromatographic deprivation was done with Methanol extract.

Densitometry scanning of the HPTLC pattern showed 4 spots corresponding to hRf values $43.90,3.35,32.69,20.06$. In short wave UV $254 \mathrm{~nm}$ and 3 spots corresponding to hRf values 36.01, 5.34, 58.66 , obtained in long wave UV $366 \mathrm{~nm}$. Though it may not be able to identify particular chemical constituent from the spots obtained, the pattern may be used as a reference standard for further quality control researches (Figure 6).

Vasavaleha: Vasavaleha is a traditional Ayurvedic oral Herbal formulation consisting of five herbs, Vasaka (Adhatoda vasica Nees.), Pippali (Piper longum Linn.), Sugar, Ghee and Honey. It is available as a popular proprietary, from most manufacturers of ayurvedic drugs [20]

HPTLC: A selective, precise and accurate High Performance Thin Layer Chromatography (HPTLC) method has been developed for the simultaneous quantification of Vasicine and Piperine in Vasavaleha 
Citation: Garg S, Mishra A, Gupta R (2013) Fingerprint Profile of Selected Ayurvedic Churnas/Preparations:An Overview. Altern Integ Med 2: 125. doi:10.4172/2327-5162.1000125

Page 5 of 10

\begin{tabular}{|c|c|c|c|c|}
\hline S. No. & Ayurvedic preparations/extract & Constituents & Solvent system and Scanning wavelength & Standard \\
\hline 1. & $\begin{array}{l}\text { Ashwagandha capsule } \\
\text { (methanol) [47] }\end{array}$ & Withania somnifera & $\begin{array}{l}\text { HPTLC: Chloroform: Methanol }(9: 1) \text { at } 254 \text { and } \\
366 \mathrm{~nm} \text {. }\end{array}$ & Not mentioned \\
\hline \multirow[t]{3}{*}{2.} & $\begin{array}{l}\text { Ashtaangaavaleha } \\
\text { (methanol) [21] }\end{array}$ & $\begin{array}{l}\text { Myrica negi, Inula racemosa, Pistacia integerrima, } \\
\text { Ptychotis ajawan, Carum carvi } \\
\text { Zinziber officinale, Piper nigrum, Piper longum, } \\
\text { Saccharum officinarum, honey }\end{array}$ & $\begin{array}{l}\text { HPTLC:-Toluene:Ethyl acetate } \\
(9: 1 v / v) . \text { at } 254 \text { and } 366 \mathrm{~nm} .\end{array}$ & Not mentioned \\
\hline & $\begin{array}{l}\text { Ashwagandharishta } \\
\text { (methanol) [41] }\end{array}$ & Withania somnifera & $\begin{array}{l}\text { HPTLC:-Toluene:ethylacetate: formic acid at } \\
540 \mathrm{~nm} \text {. }\end{array}$ & $\begin{array}{l}\text { Beta-sitosterol D } \\
\text { glucoside }\end{array}$ \\
\hline & $\begin{array}{l}\text { Ashwagandha vati } \\
\text { (methanol) [41] }\end{array}$ & Withania somnifera & $\begin{array}{l}\text { HPTLC:-Toluene:ethylacetate: formic acid at } \\
540 \mathrm{~nm} \text {. }\end{array}$ & $\begin{array}{l}\text { Beta-sitosterol D } \\
\text { glucoside }\end{array}$ \\
\hline 5. & $\begin{array}{l}\text { Amritaprasa ghrita, brahmi ghrita, } \\
\text { chagalyadi ghrita and phala ghrita } \\
\text { (hexane) [48] }\end{array}$ & Not mentioned & $\begin{array}{l}\text { HPLC:-Hexane-isopropanol at } 220 \mathrm{~nm} \text {. injection } \\
\text { volume: } 20 \mu \mathrm{l} \text { and flow rate were } 2 \mathrm{ml} / \mathrm{min}\end{array}$ & Not mentioned \\
\hline 6. & $\begin{array}{l}\text { Arkavati, Krvyadras and } \\
\text { Marichyadi taila } \\
\text { (methanol) [49] }\end{array}$ & Mainly Piper nigrum & $\begin{array}{l}\text { HPLC:-Methanol: water in the volume ratio of } \\
70: 30 \text { at } 342 \mathrm{~nm} \text {. at a flow rate of } 1.0 \mathrm{~mL} \text { min-1 }\end{array}$ & Piperine \\
\hline 7. & $\begin{array}{l}\text { Polyherbal Acnovin capsule } \\
\text { (aq., methanol, ethyl acetate) [50] }\end{array}$ & $\begin{array}{l}\text { Mahamajishthadi kwath, Panch neem } \\
\text { churna, Sariva, Sonamukhi, Khadir twak, Haridra, } \\
\text { Amla, Bibhitaki, Haritaki and Gandhak rasayana }\end{array}$ & $\begin{array}{l}\text { HPTLC:-Water: acetonitrile: glacial acetic acid } \\
(9: 1: 0.2) \text { at } 540 \mathrm{~nm} \text {. } \\
\text { HPLC:-Water: acetonitrile: glacial acetic acid } \\
(9: 1: 0.2) \text { at } 272 \mathrm{~nm} \text {. At flow rate } 1 \mathrm{ml} / \mathrm{min} \text {. }\end{array}$ & Gallic acid \\
\hline 8. & $\begin{array}{l}\text { Chyawanprash } \\
\text { (aqueous and methanol) [22] }\end{array}$ & $\begin{array}{l}\text { Dashmula, Ashtavarga, chaturjata class herbs, } \\
\text { mainly Embllica officinalis. Piper longum Termenalia } \\
\text { chebula, Cinnamomum zeylanicum, Elettaria } \\
\text { cardamomum, honey etc. }\end{array}$ & $\begin{array}{l}\text { HPTLC:-Toluene: ethyl acetate: formic acid: } \\
\text { ethanol }(6: 4: 0.3: 0.4) \text { at } 254 \text { and } 366 \mathrm{~nm} \text {. }\end{array}$ & $\begin{array}{l}\text { piperine, catechin, } \\
\text { epicatechin and } \\
\text { gallic acid }\end{array}$ \\
\hline 9. & $\begin{array}{l}\text { Ikshvadi avaleha } \\
\text { (methanol) [19] }\end{array}$ & $\begin{array}{l}\text { Phyllanthus urinaria, Saccharum officinarum, } \\
\text { Bambusa arundinacea, Mucuna prurita, Pipernigrum, } \\
\text { Cinnamomum zeylanicum, Elettaria cardamomum, } \\
\text { honey }\end{array}$ & $\begin{array}{l}\text { HPTLC:-Toluene:Ethyl acetate }(7: 3 \mathrm{v} / \mathrm{v}) \text {. at } 254 \\
\text { and } 366 \mathrm{~nm} \text {. }\end{array}$ & Not mentioned \\
\hline \multirow[t]{2}{*}{10.} & $\begin{array}{l}\text { Krimimudgara rasa } \\
\text { (ethanol) [51] }\end{array}$ & $\begin{array}{l}\text { Apium graveolens, Embellia ribes, Strychnous } \\
\text { Nux vomica and Butea monosperma, Rasa (Mercury) } \\
\text { and gandhaka (Sulphur) }\end{array}$ & $\begin{array}{l}\text { HPTLC:-n-Propanol: } n \text { Butanol: } 4 \text { N Ammonia } \\
(7: 1: 2) \text {, Toluene: Ethyl acetate: Di-ethylamine } \\
(7: 2: 1) \text { at } 254 \text { and } 366 \mathrm{~nm} \text {. }\end{array}$ & $\begin{array}{l}\text { Embelin, } \\
\text { strychnine }\end{array}$ \\
\hline & $\begin{array}{l}\text { Kalmegh containing asava } \\
\text { (methanol and di-ethyl ether) [52] }\end{array}$ & Andrographis peniculata & HPTLC:-Benzene:ethyl acetate $(5: 5)$ at $222 \mathrm{~nm}$. & Andrographolide \\
\hline 12. & $\begin{array}{l}\text { Punarnavashtak kwath } \\
\text { (methanol) [53] }\end{array}$ & $\begin{array}{l}\text { Boerhaavia diffusa, Picrorhiza Kurroa, Tinospora } \\
\text { cordifolia, Zingiber officinalis, Berberis aristata, } \\
\text { Terminalia chebula, Azadirachta indica. and } \\
\text { Tricosanthes dioica }\end{array}$ & $\begin{array}{l}\text { toluene:ethylacetate:methanol:formic acid } \\
(3: 3: 0.2: 0.8) \text { and scanned at } 366 \mathrm{~nm} \text { for } \\
\text { berberine and at } 280 \mathrm{~nm} \text { for gallic acid }\end{array}$ & $\begin{array}{l}\text { Gallic acid and } \\
\text { berberine }\end{array}$ \\
\hline 13. & $\begin{array}{l}\text { Sanjivani Vati } \\
\text { (diethyl ether) [54] }\end{array}$ & $\begin{array}{l}\text { Embelia ribes, zingiber officinalis, Terminalia } \\
\text { chebula, Terminalia belerica, Tinospora cordifolia, } \\
\text { Aconitum heterophyllum, Emblica officinalis }\end{array}$ & $\begin{array}{l}\text { HPLC:-Acetonitrile: water }(20: 80) \text {, acetonitrile: } \\
\text { water: acetic acid }(48: 52: 1) \text { at } 254 \mathrm{~nm} \text { at flow } \\
\text { rate } 1 \mathrm{ml} / \mathrm{min} \text {, injected vol. } 10 \mu \mathrm{l}\end{array}$ & Embelin, piperine \\
\hline 14. & $\begin{array}{l}\text { Triphala Mashi } \\
\text { (methanol) [55] }\end{array}$ & $\begin{array}{l}\text { Terminalia chebula, Terminalia belerica, Phyllanthus } \\
\text { emblica }\end{array}$ & $\begin{array}{l}\text { Terminalia chebula, Terminalia belerica, } \\
\text { Phyllanthus emblica }\end{array}$ & Gallic acid \\
\hline 15. & $\begin{array}{l}\text { Triphalaguduchyadi Vati } \\
\text { (methanol) [56] }\end{array}$ & $\begin{array}{l}\text { Terminalia chebula, Terminalia belerica, Emblica } \\
\text { officinalis, Cyperus rotandus, Tinospora cordifolia }\end{array}$ & $\begin{array}{l}\text { HPTLC:-Toluene:Ethylacetate: Acetic acid }(7: 2: 1) \\
\text { at } 254 \text { and } 366 \mathrm{~nm} .\end{array}$ & Not mentioned \\
\hline 16. & $\begin{array}{l}\text { Triphla capsule } \\
\text { (Methanol) [57] }\end{array}$ & $\begin{array}{l}\text { Terminalia chebula, Terminalia belerica, Phyllanthus } \\
\text { emblica }\end{array}$ & HPTLC:-Chloroform: Methanol (7:3) at $540 \mathrm{~nm}$ & Gallic acid \\
\hline 17. & $\begin{array}{l}\text { Vasavaleha } \\
\text { (chloroform and methanol) [20] }\end{array}$ & $\begin{array}{l}\text { Vasaka (Adhatoda vasica Nees.), Pippali (Piper } \\
\text { longum Linn.), Sugar, Ghee and Honey. }\end{array}$ & $\begin{array}{l}\text { HPTLC:-Dioxane:Toluene: Ethylacetate: Methanol: } \\
\text { Ammonia }(1.5: 2: 1: 1: 0.3 \% \mathrm{v} / \mathrm{v}) \text { at } 285 \mathrm{~nm} .\end{array}$ & $\begin{array}{l}\text { Vasicine and } \\
\text { Piperine }\end{array}$ \\
\hline 18. & $\begin{array}{l}\text { Vyaghrihareetaki avaleha } \\
\text { (acid hydrolysed Methanol } \\
\text { extract) [18] }\end{array}$ & $\begin{array}{l}\text { Solanum xanthocarpum, Terminalia chebula, } \\
\text { Piper nigrum, Piper longum, Zingiber officinale, } \\
\text { Cinnamomum zeylanicum, Cinnamomum tamala } \\
\text { Elettaria cardamomum, Mesua ferrea, honey, } \\
\text { jaggery }\end{array}$ & $\begin{array}{l}\text { HPTLC:-Toluene:Ethyl acetate :Glacial acetic } \\
\text { acid: Formic acid (5:5:1:0.5) at } 254 \text { and } 336 \mathrm{~nm} \text {. }\end{array}$ & Not mentioned \\
\hline 19. & $\begin{array}{l}\text { Vatari guggulu } \\
\text { (n-hexane) [58] }\end{array}$ & $\begin{array}{l}\text { Commiphora wightii, Ricinus communis, Terminalia } \\
\text { chebula, Terminalia belerica, Emblica officinalis, } \\
\text { Sulphur }\end{array}$ & HPTLC:-Toluene: acetone $(9: 1)$ at $250 \mathrm{~nm}$. & Not mentioned \\
\hline 20. & $\begin{array}{l}\text { Yogaraja guggulu } \\
\text { (aq. and methanol) [59] }\end{array}$ & $\begin{array}{l}29 \text { ingredients: Plumbago zeylanica, Piper longum, } \\
\text { Hyosymus niger, Commiphora mukul, Ricinus } \\
\text { communis, Terminalia chebula, Terminalia belerica, } \\
\text { Embelia ribes etc. }\end{array}$ & HPLC:-Petroleum ether: Ethyl acetate & Not mentioned \\
\hline
\end{tabular}

Table 3: Fingerprint profile of other Ayurvedic preparations.

(chloroform and methanol extract) as well as its bulk drug.

The method employed TLC aluminum plate precoated with silica gel $60 \mathrm{~F} 254$ as a stationary phase. The solvent system consists of Dioxane: Toluene: Ethyl acetate: Methanol: Ammonia (1.5:2:1:1:0.3 $\% \mathrm{v} / \mathrm{v})$. This system was found to give compact spot for Vasicine and Piperine. Densiometric analysis was carried out in the absorbance mode at $285 \mathrm{~nm}$. The linear regression analysis data for the calibration plot showed good linear relation with $\mathrm{r}^{2}=0.992$ and 0.993 with respect to peak area for Vasicine and Piperine respectively, in concentration range $2-10 \mu \mathrm{g} /$ spot (Figure 7 ).

The data generated indicate that Vasavaleha contains a number of markers that may have a prominent role to play, for the therapeutic 

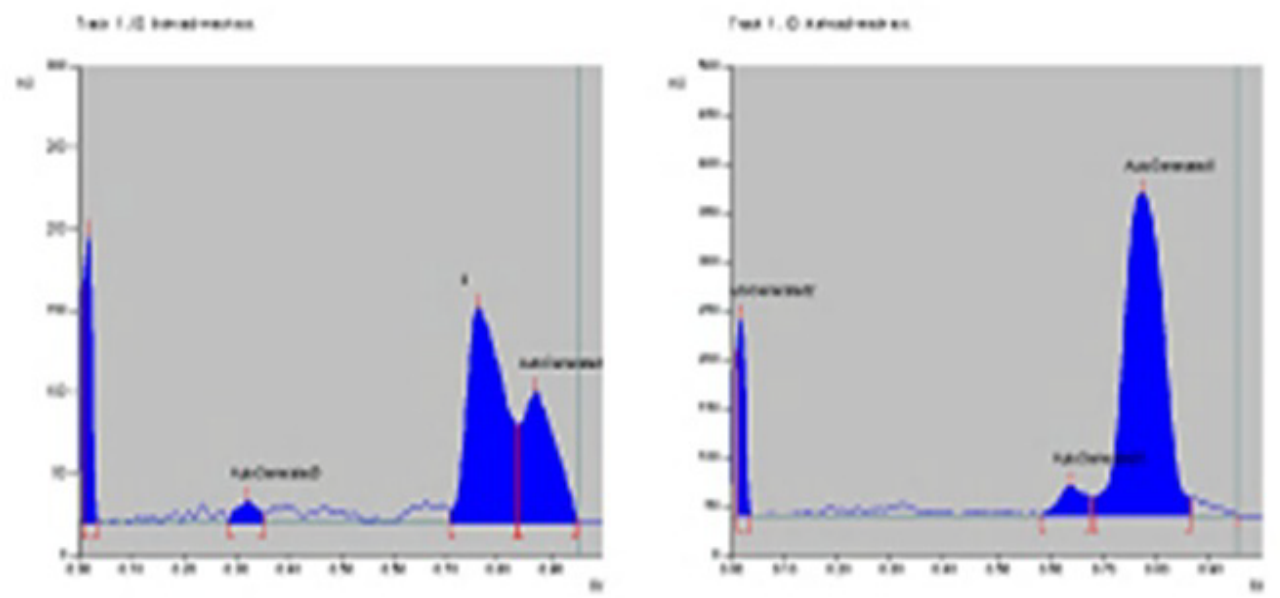

Figure 6: Densitogram of Ikshvadi Avaleha at 254 and $366 \mathrm{~nm}[19]$.
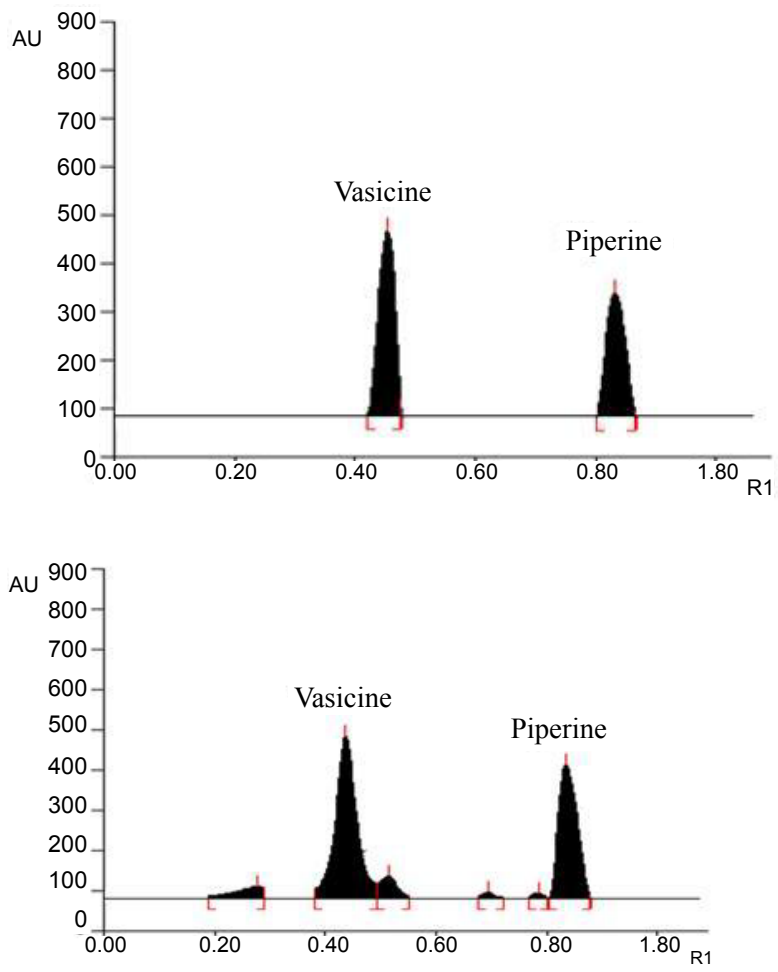

Figure 7: HPTLC Chromatogram of standard Vasicine and Piperine and HPTLC Chromatogram of Vasavaleha [20].

activity. The proposed HPTLC methods for simultaneous estimation of Vasicine and Piperine from Vasavaleha, seems to be accurate, precise, reproducible and repeatable. It is the first attempts, when both the markers in Vasavaleha were simultaneously estimated and compared for the respective raw materials.

Ashtaangaavaleha: Ashtaangaavaleha is indicated for the management of Jwara (Fever), Kaasa (Cough), Swaasa (Dyspnoea/ Asthma), Aruci (Tastelessness) and Chardi (Emesis). There has been an

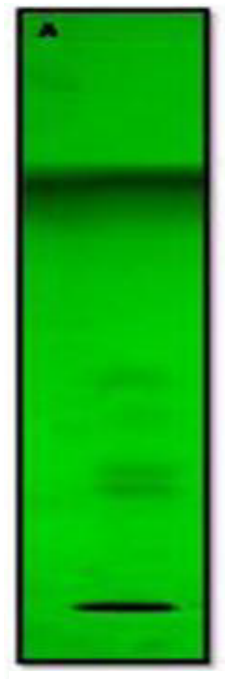

$\mathrm{A}=\mathrm{Z64} \mathrm{nm}$

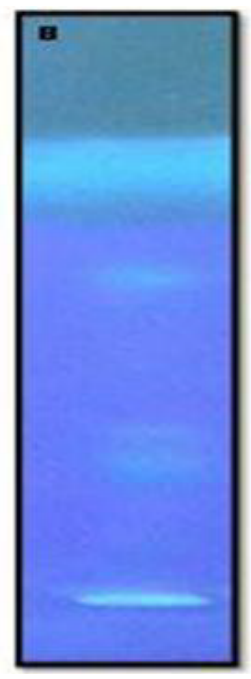

$B=389 \mathrm{~nm}$

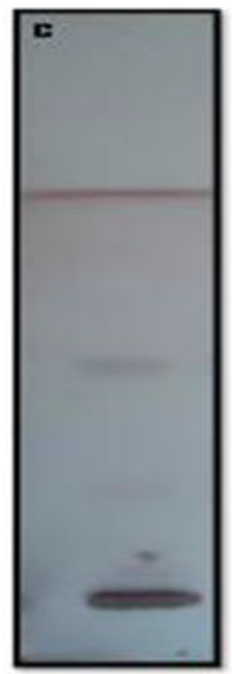

C=after spray
Figure 8: HPTLC of Ashtaangaavaleha (Methanol Extract) [21].

increase in demand for the Phyto-pharmaceutical products of Ayurveda so a new pharmaceutical preparation in the form of Ashtaangaavaleha was tried to standardize which is economical in terms of time and machinery usage. The phytochemical analysis and High Performance Thin Layer Chromatography has been performed to confirm its identity, quality and purity [21].

High performance thin layer chromatography (HPTLC) study: In High performance thin layer chromatography (HPTLC) study of ashtaangavaleha (methanol extract) using Toluene: Ethyl acetate (9:1v/v), visual observation under UV light showed few spots, but on analyzing under densitometer much more was observed. Chromatogram shows 8 prominent spots at Rf $0.02,0.13,0.22,0.32,0.49,0.56,0.77,0.94$ in short wave UV254 $\mathrm{nm}$ and 5 prominent spots at Rf $0.02,0.20,0.49$, $0.56,0.65$ in long wave UV $366 \mathrm{~nm}$. Details are noted in the Table 1 and Figure 2. Then the plate was sprayed with Anisaldehyde sulphuric acid 
followed by heating and then visualized in day light shows 3 prominent spots at Rf 0.18, 0.36, 0.55 (Figure 8).

Chyawanprash: Chyawanprash is a traditional polyherbal formulation, which is widely used as rejuvenator, anabolic, immunomodulator and memory enhancer. Chyawanprash contains the pulp of Embelica officinalis as the prime ingredient, along with powder and extract of several other herbs [22].

HPTLC: HPTLC analysis of aqueous and methanol extract was performed using toluene: ethyl acetate: formic acid: ethanol (6:4:0.3:0.4) for developing finger print profile of piperine, catechin, epicatechin and gallic acid at 254 and $366 \mathrm{~nm}$.

\section{Asava and arishta}

Asavas and arishtas are very popular in India, probably due to their taste and alcoholic content in addition to their medicinal uses and physiological importance [23]

These are fermented preparations of medicinal plants. The fermentation procedure adopted to prepare these preparations is termed as 'Sandhaana kalpanaa' and the ferment used to stimulate fermentation is termed as 'Sandhaana dravya'. Arishtas are usually prepared by fermenting expressed juice ('swarasa'), whereas 'Asavas' are prepared from fermentation of decoction (Kwaatha). Sugar or jaggery and powders (choorna) of other medicinal plants as required along with a natural ferment are added to these two liquids and they are left in a closed container till the fermentation is completed. Aasava and Aristaas can be prepared from 'swarasa' or 'kwaatha' (as the case may be) of single plant or from a mixture of 'swarasa' or 'kwaatha' from multiple plants. This facilitates the extraction of the active principles contained in the drugs [8].

Arjunarishta: Arjunarishta (Parthadyarishta) is an important Ayurvedic formulation used for cardiovascular disorders and is prepared by fermenting the decoction of specified plant materials using flowers of Woodfordia fruticosa [23].

An HPLC-PDA method was developed for the standardization of Arjunarishta by quantitative estimation of major antioxidant compounds, ellagic acid, gallic acid, ethyl gallate, quercetin and kaempferol as markers.

HPLC: HPLC method was developed for the formulation after several trials for separation of phenolic acids and flavonoids. The flavonoids showed very high retention time ( $>75$ minutes) with the reported method. In the present study, a shorter run time (45 min) was achieved with gradual increase of organic phase (acetonitrile). Five phenolic compounds were identified in Arjunarishta; these were gallic acid, ethyl gallate, ellagic acid, quercetin and kaempferol. The chromatogram also showed several other unidentified peaks. A binary gradient system consisting of water-acetonitrile-acetic acid as mobile phase was able to separate these compounds (Figure 9).

Chandanasava: Chandanasava is one of ancient, commonly used Ayurvedic formulations. The herbal formulation is made up of Santalum album and other 24 plant ingredients. Chandanasava is prescribed for treatment of karsya (malnutrition), sukramehe (presence of semen in urine), mutrakrcchra (painful micturation), hrdroga (heart diseases), agnimandya (loss of appetite) [24].

HPTLC: Fingerprinting of different extracts (petroleum ether, dichloromethane, ethyl acetate) was done by using selected solvent system pet. Ether: ethyl acetate (9.5:0.5), pet. Ether: ethyl acetate (9:1),
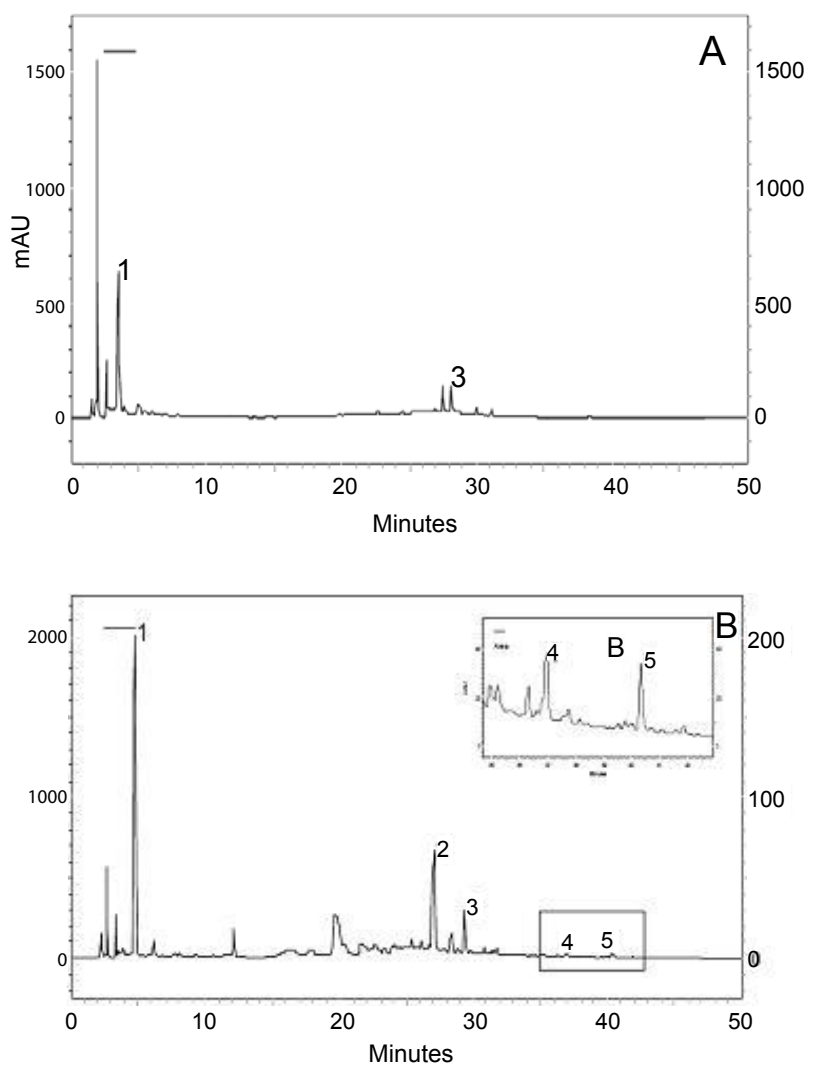

Figure 9: Chromatogram of A) Decoction; B) Arjunarishta at $280 \mathrm{~nm} .1$ Gallic acid, 2. Ethyl gallate, 3. Ellagic acid, 4. Quercetin, 5. Kaempferol [23].

toluene: ethyl acetate: acetic acid:water (3:3:0.8:0.2 v/v) respectively for extracts, visualised at $366 \mathrm{~nm}$ and chromatogram was scanned with spectrodensitometer.

\section{Tablet/Vati}

Vati and Gutika-These are in the herbal preparation in the form of tablets or pills made of one or more drugs of plant or mineral origin and these too comprise other several items [25]. In Ayurveda there are several other different type of formulation like Vatis-Gutika (Pills), Rasa yoga (mineral based herbal formulation), Tailas (oil based herbal formulation), Guggulu etc.

Amalant tablet: Each tablet contains Embelica officinalis $201 \mathrm{mg}$ and 15 other ingredients. Amalant offers a multi-pronged approach in the treatment of hyperacidity and acid peptic disorders [26]

\section{HPTLC of gallic acid}

Mobile phase for Gallic Acid is Toluene: Ethyl Acetate: Formic Acid (6:3:1 v/v/v), and Scanning wavelength: $254 \mathrm{~nm}$, Mode of scanning: Absorption [Deuterium], Standard: Gallic acid $0.1 \mathrm{mg} / \mathrm{ml}[10 \mu \mathrm{l}]$.

The Rf value of Standard Gallic Acid was found to be 0.34 and peak area 5097.0. Amalant Tablet extract showed nine peaks, the fourth peak $\mathrm{Rf}$ value (0.34) was coinciding with standard $\mathrm{Rf}$ value and its area calculated was 249.71 at $100 \mu \mathrm{g} / \mathrm{ml}$ of standard and sample concentration, The amount of gallic acid was found to be $4.89 \%$ (Figure 10).

Sulaharan yoga: Sulaharan Yoga (SY), an Ayurvedic polyherbal 

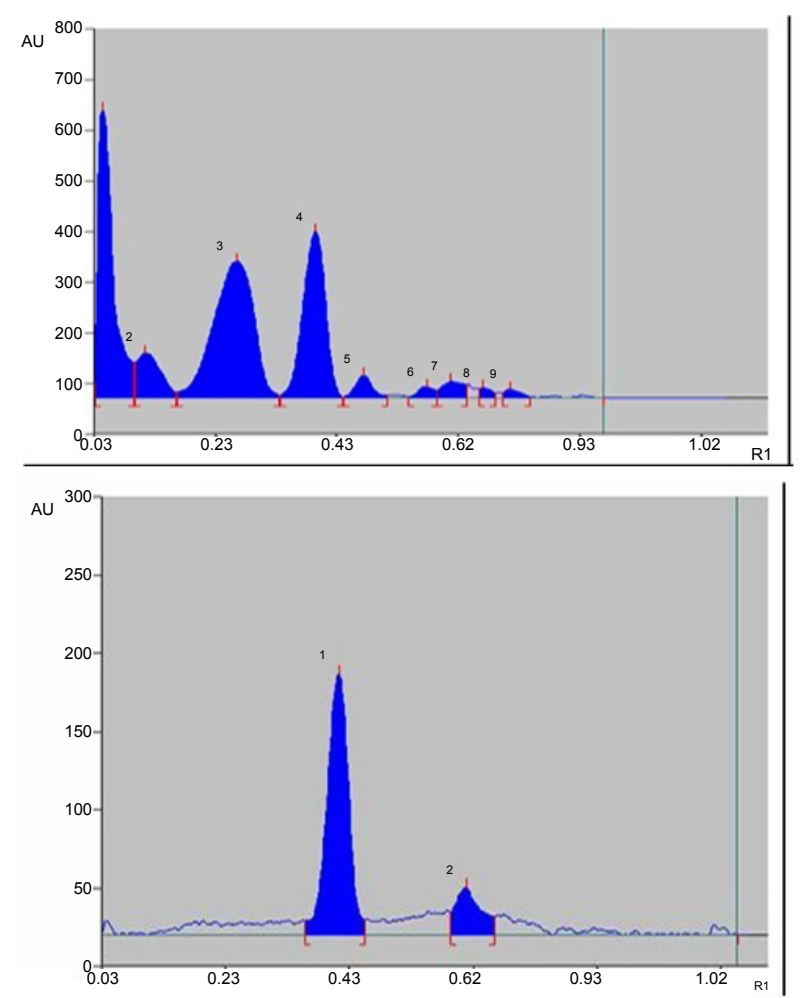

Figure 10: HPTLC Chromatogram Of Amalant Tablet Extract \& Gallic Acid [26].
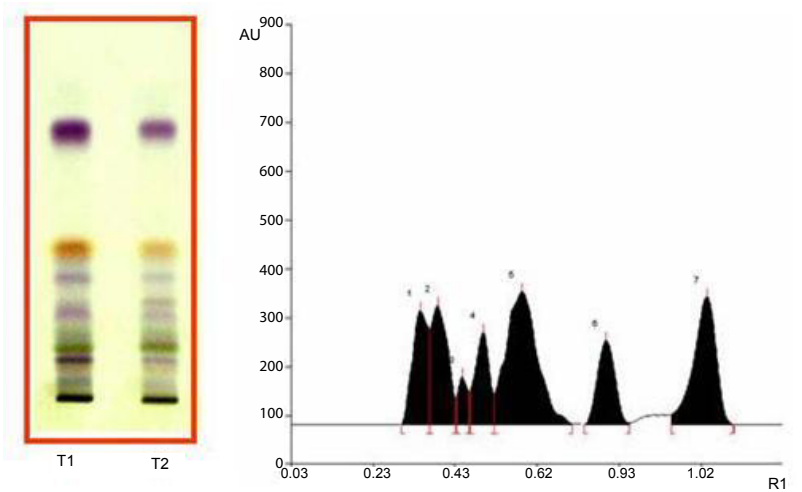

Figure 11: TLC fingerprint profile of formulations, T1: Sulaharana Yoga (Inhouse); T2: Sulaharana Yoga (Sharmayu); SolventSystem: Toluene: ethyl acetate: formic acid $(5: 15: 0.5)$ \& HPTLC fingerprinting of sulaharan yoga [27].

formulation, consists of Strychnos nux-vomica and other seven ingredients in Vati (Tablet) form, 1. Terminalia chebula (Family: Combretaceae, part: dried fruit), 2. Zingiber officinale (Family: Zinglberaceae, part: dried rhizome), 3. Piper nigrum (Family: Piperaceae, part: dried fruit), 4. Piper longum (Family: Piperaceae, part: dried fruit), 5. Strychnos nux-vomica (Family: Fabaceae, part: dried seed), 6. Ferula foetida (Family: Umbelliferae, part: oleo-gumresin), 7.Sulphur and 8. Rock salt (Saindhava lavana) [27].

HPTLC: HPTLC study of extracts (methanolic) of the separate ingredients, formulation (laboratory scale) and formulation (commercial scale) were carried out using the different biomarker compounds corresponding to the therapeutically active ingredients to ensure the presence of active ingredients in all the formulations. HPTLC fingerprint profile of an ayurvedic Sulaharan Yoga formulations are depicted in figure represents the presence of all major ingredients in proportional quantity in the formulations, in absence of any impurities. This confirms the consistency in the batches of the laboratory scale preparation and commercial scale (Figure 11).

From superimposition study a band (Rf 0.44) corresponding to Gallic acid is visible in both Terminalia chebula and Sulaharana yoga formulations, indicate the presence of Terminalia chebula in the formulations.

It is generally believed that for monitoring quality control parameters, HPTLC fingerprinting is an ideal option which involves comparative parameter between a standard and a test sample. The use of biomarkers ensures that the concentration and ratio of components in the herbal mixture are present as per claims and also in in reproducible levels in raw materials batches and in the final dosage form batches. In this way use of markers and chromatographic fingerprinting technique can give useful information assisting manufacturing control,minimising variations in production batches and assuring batch to batch consistency, with reproducible results [28].

Nisha amalaki vati: Nisha Amalaki Vatti is a polyherbal formulation containing Curcuma longa and Phyllanthus emblica used as anti-diabetic agents marketed by Ayush, India [29].

Standardisation by UV, HPLC and HPTLC method has been studied for the simultaneous analysis of curcuminoids and gallic acid in combined polyherbal formulation. The proposed method was found to be simple, sensitive, accurate, precise, economical and rapid for the routine simultaneous estimation of these two phytoconstituents in a combined dosage form. The value of the standard deviation and coefficient of variation were satisfactory. In the simultaneous equation method wavelength of respective absorbance maxima i.e. $227 \mathrm{~nm}$ for gallic acid and $427 \mathrm{~nm}$ for curcuminoids were used for the analysis of the phytoconstitution in the standard and tablet (Figure 12).

\section{Conclusion}

Fingerprint profile is quite helpful in setting up of standards and thus to keep a check on intentional/unintentional adulteration. The present review is an attempt to compile the major studies carried out on Ayurvedic Churnas/prepapations, which may be of use to develop/ compile the fingerprint profile for evaluating the purity and quality of churnas/preparations, thus helpful as a reference in developing

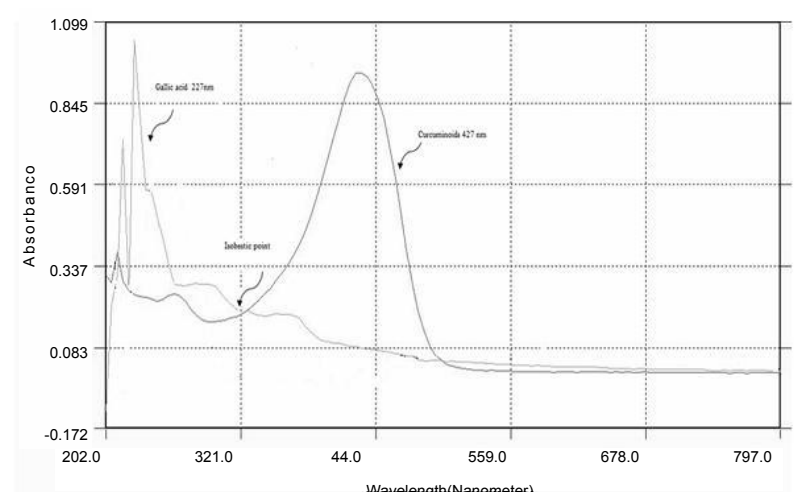

Figure 12: Over lapping spectra showing Curcuminoids and Gallic acid [28] 
pharmacopoeial standards, present compilation would also be helpful to overcome batch to batch variations in traditional preparation of different Ayurvedic churna/preparations.

\section{References}

1. Rathi A, Rai V, Khatoon S, Rastogi S, Rawat AKS, et al. (2010) Evaluation of Ayurvedic compound formulation III-Laghugangadhar Churna. Indian J Traditional Knowledge 9: 576-580.

2. Waghmare PR, Kochar NI (2011) Quality control of Varuna Kwatha Churna, an Ayurvedic formulation and its comparative study with marketed formulations. Int J Pharm Sci Res 2: 1861-1868.

3. Gupta V, Jain UK (2011) Status of piperine content in Ayurvedic formulation: method standardization by HPTLC. Res J Pharm Biol Chem Sci 2: 524-531.

4. Mukherjee PK (2005) Quality Control of Herbal Drugs. New Delhi: Business Horizons 741

5. Ong ES (2004) Extraction methods and chemical standardization of botanicals and herbal preparations. J Chromatogr B Analyt Technol Biomed Life Sci 812 23-33.

6. Guideline on Validation of Analytical Procedure- Methodology (1996) Geneva: International Conference on Harmonization.

7. Meena AK, Rao MM, Panda P, Kiran, Yadav A, et al. (2010) Standardisation of Ayurvedic polyherbal formulation, Pancasama Churna. Int J Pharmacog Phytochem Res 2: 11-14.

8. Ayurvedic Pharmacopoeia of India (2007) (1st edn) New Delhi: The Controller of Publications Civil Lines 1: 38.

9. The Ayurvedic Formulary of India-Part-I (2000 ) (2nd edn) New Delhi: Government of India, Ministry of Health and Family Welfare 31.

10. The Ayurvedic Pharmacopoeia of India (2001) (1st edn) New Delhi: Government of India, Ministry of Health and Family Welfare 1: 162.

11. Gautam A, Yadav K, Shah J (2011) Formulation and evaluation of Chaturjat Churna: containing 4 ingredients. Int J Pharm Life Sci 2: 554-558.

12. Sitapara N, Buch P, Dudhrejia A, Sheth NR (2011) Standardization of Caturjata Churna-An Ayurvedic Polyherbal Formulation. IJPI'S J Pharmacog Herbal Formulations 1: 57-62.

13. Mali DS, Kondawar MS, Kamble KG (2011) Quantitative estimation of Gallic acid and Ascorbic acid in a marketed herbal medicine: Triphala Churna by High Performance Thin Layer Chromatography. Int J Pharm Tech Res 3: 1593 1599

14. Jain V, Vyas A, Saraf S (2011) TLC Densitometric Methods for Quantification of Gallic Acid in Triphala Churna for Routine Quality Control. Res J Pharm Tech 4: 230 .

15. Shailajan S, Sayed N, Tiwari B, Harshvardhan J (2011) Standardization of an ayurvedic formulation: Trikatu churna using bioanalytical tools. Int $\mathrm{J}$ Res Ayurveda Pharmacy 2: 1676-1678.

16. Vyas A, Jain V, Singh D, Shukla SS, Saraf S (2011) TLC Densitometric Method for the Estimation of Piperine in Ayurvedic Formulation Trikatu Churna. Oriental J Chem 27: 301-304.

17. Mahajan DA, Pai RN (2011) Development and Validation of HPLC Method for Quantification of Phytoconstituents in Haritaki Churna. Int J ChemTech Res 3: 329-336.

18. Roshy JC, Biswajyoti P, Shukla VJ, Harisha CR (2011) Pharmacognostical and Analytical study of Vyaghrihareetaki avaleha: An Ayurvedic polyherbal formulation. J Pharmacy Res 4: 3575-3577.

19. Gohel SD, Patel KS, Harisha SR, Shukla VJ (2011) Pharmacognostical \& Pharmaceutical Evaluation of Ikshvadi Avaleha. J Pharm Biomed Sci 11: 1-4.

20. Patel RK, Kanani RJ, Patel VR, Patel MG (2010) Development and Validation of HPTLC Method for Simultaneous Quantification of Vasicine and Piperine in Vasavaleha. Int J Pharmaceut Res 2: 14-17.

21. Dubey AK, Rajagopala S, Patel KS, Harisha CR, Shukla VJ (2011) Pharmacognostical and phyto-chemical standardization of ashtaangaavaleha: a polyherbal formulation. Pharmacie Globale Int $\mathrm{J}$ comprehensive pharmacy 11: $1-4$

22. Kasar RP, Laddha KS, Shukla A (2006) Development of quality control methods for polyherbal formulation, Chyawanprash. Nat Prod Rad 5: 33-41.

23. Lal UR, Tripathi SM, Jachak SM, Bhutani KK, Singh IP (2009) HPLC Analysis and Standardization of Arjunarishta - An Ayurvedic Cardioprotective Formulation. Sci Pharm 77: 605-616.

24. Katekhaye S, Singh A (2012) Standardisation of Ayurvedic polyherbal formulation: Chandanasava. Int J Res Ayurveda Pharmacy 2: 665-669.

25. Lather A, Gupta V, Bansal P, Singh R, Chaudhary AK (2010) Pharmacological Potential of Ayurvedic Formulation: Kutajghan Vati- A Review. J Adv Sci Res 1: $41-45$

26. Patel NV, Telange DR (2011) Qualitative and Quantitative Estimation of Gallic Acid and Ascorbic Acid in Polyherbal Tablets. Int J Pharm Sci Res 2: 23942398.

27. Pattanaya P, Jena RK, Panda SK (2010) HPTLC fingerprinting in the standardization of sulaharan yoga: an ayurvedic tablet formulation. Int $\mathrm{J}$ Pharmaceut Sci Rev Res 3: 33-36.

28. Pattanayak P, Mohapatra P, Jena RK, Panda SK (2011) Standardization of sulaharan yoga: an ayurvedic tablet formulation. Indian J Pharm Sci 73: 65-70.

29. Rubesh KS, Kishan RJ, Rao VKN, Duganath N (2010) Simultaneous Spectrophotometric Estimation of Curcuminoids and Gallic Acid in Bulk Drug and Ayurvedic Polyherbal Tablet Dosage Form. Int J Pharmaceut. Quality Assurance 2: 56-59.

30. Patra KCH, Suresh $P$ (2009) Standardization of polyherbal siddha formulation, Amukkara Choornam. Ind J Traditional Knowledge 8: 449-452.

31. Verma JK, Joshi AV (2006) Rapid HPTLC method for identification and quantification of curcumin, piperine and thymol in an ayurvedic formulation. $J$ Planar Chromatography - Modern TLC 19: 398-400.

32. Prasad GP, Trimurtulu G, Reddy KN, Naidu ML (2010) Analytical study of Kuberaksha/Kantaki Karanja Patra Churna [Caesalpinia Bonduc (L.) Roxb. leaf powder]. Ayu 31: 251-254

33. Kumar PS Kumar VK (2011) Standardization of a polyherbal Ayurveda formulation, Nisamalaki Churna tablet. J Pharm Res 4: 1483-1487.

34. Priyanka S, Vishal S, Shyam S (2010) Standardization of Ayurvedic formulation, Panchsakar Churna. J Global Pharma Technol 2: 46-50.

35. Pattanayak P, Behera M, Mohapatra P, Panda SK (2011) Standardization and Evaluation of Laxative Activity of a Poly Herbal Formulation. Scholar Research Library Der Pharmacia Lettre 3: 276-286.

36. Rastogi S, Khatoon S. Pandey MM (2008) Evaluation of Ayurvedic compound formulation 2- Palas abijadi churna. Indian J Traditional Knowledge 7: 384 388.

37. Mistry H, Shukla H, Prajapati N (2010) Standardization of Panchkol churna by HPTLC. J Planar Chromatography 105: 126.

38. Patra KC, Pareta SK Singh B (2011) Comparative standardization of a Polyherbal Ayurvedic formulation Talishadi churna. Indian J. Traditional Knowledge 10: 608-611.

39. Sudani RJ, Akbari BV, Vidyasagar G (2011) Development and Validation of HPTLC Method for Simultaneous Quantitation of Embelin and Assay of Marketed Formulation. Int J Pharm Biol Archives 2: 652-656.

40. Kaur R, Meena AK, Singh B, Rao MM (2010) Evaluation of Standardization parameters of Ashwagandha Churna-Ayurvedic drug. J Pharmacy Res 3: 831 832.

41. Tatke P, Supriya J, Gabhe SY (2010) Marker based standardization of commercial formulations and extracts containing beta-sitosterol using HPTLC. Int J Res Ayur Pharmacy 1: 616-623.

42. Shukla K, Saraf S (2008) Development of fingerprints for an Ayurvedic formulation Bhaskar Lavan churna via Piperine estimation by High Performance Liquid Chromatography. Indian J Pharm Educ Res 42: 127-132.

43. Pandey RK, Saraf S (2010) Development of fingerprint for single component analysis of an Ayurvedic formulation (Sitopaladi Churna) by High Performance Liquid Chromatography. Scholar Research Library Der Pharmacia Lettre 2 : 464-470.

44. Jain V, Vyas A, Saraf S (2011) HPLC Determination of Piperine in 'Trikatu Churna' a Potent Ayurvedic Formulation for Routine Quality Control. Asian J Res Chem 4: 183. 
Citation: Garg S, Mishra A, Gupta R (2013) Fingerprint Profile of Selected Ayurvedic Churnas/Preparations:An Overview. Altern Integ Med 2: 125. doi:10.4172/2327-5162.1000125

45. Bais SK, Chandewar AV (2011) Comparative evaluation of Endosulfan content in triphala churna marketed in Yavatmal district of India by HPLC method. In J Pharm Pharm Sci 3: 3540.

46. Sudani RJ, Akbari BV, Vidyasagar J. Sharma P (2011) Quantitative and Chromatographic Fingerprint Analysis of Embelia ribes churna Formulations by HPLC Method. Int J Pharmaceut Biol Archives 2: 657-663.

47. Soni HK, Ribadiya NC, Bhatt SB, Sheth NR (2010) Evaluation of herbal formulation (capsule) containing Ashwagandha as a single herb with their nutritional value determination. Int J Applied Biol Pharmaceut Tech 1: 960-967.

48. Shaila D, Santosh MK, Chandrakumar T, Rao IS (2004) Standardization Study of Ghritas. E J Chem 1: 151-157

49. Sunita S, Menon SN, Singh A (2010) Quantitative Analysis Of Piperine From Ayurvedic Polyherbal Formulations Using Reverse Phase High Performance Liquid Chromatography. Int J Pharm Bio Sci 1: 206-218.

50. Pandya, K, Solanki B, Shah B, Parekh D, Soni H (2011) Phyto-chemical Screening \& Evaluation of Antibacterial Activity of Polyherbal Formulation. Indo-Global J Pharmaceut Sci 1: 206-218.

51. Nakve AP, Rai PD, Deokate UA, Khadabadi SS (2011) Standardization And Quality Control Evaluation of Krimimudgara Rasa Using Microscopic Studies and HPTLC. Int J Pharm Tech 3: 1537-1547.
52. Jadhao M (2010) Estimation of Andrographolide In Herbal Powder And Polyherbal Asava by HPTLC. Int J Pharm Bio Sci 1: 242-245.

53. Shah VN, Doshi DB, Shah MB, Bhatt PA (2010) Estimation of biomarkers berberine and gallic acid in polyherbal formulation punarnavashtak kwath and its clinical study for hepatoprotective potential. Int J Green Pharm 4: 296-201.

54. Parameswaran S, Mandar ND (2010) Standardization of an ayurvedic formulation: sanjivani vati. Int J Green Pharmacy 153-155.

55. Biradar SY, Sharma P, Khandelwa KR (2007) Preparation method of optimization and physiochemical evaluation of traditional formulation. Triphla mashi. Ind J Traditional Knowledge 6: 292-297.

56. Biju KR, Shukla VJ, Harisha CR, Patel KS (2011) Pharmacognostic and phytochemical evaluation of Triphalaguduchyadi vati: An effective formulation for obesity. Int J Res Ayur Pharmacy 2: 1463-1465.

57. Pandya K, Solanki B, Maniyar K, Bhatt S (2011) Natural Herbal SupplementsAn Assessment of Their Nutritional Value and Their Phytochemical Constituents. Int J Pharma Bio Sci 2: 419-438.

58. Pradhan SK, Dash NC (2011) Standardization of Vatari Guggulu-An Ayurvedic polyherbal formulation. Int J Pharma World Res 2: 1-13.

59. Simha KRG, Laxminarayana V (2008) Standardization of Yogaraja guggulu-an ayurvedic polyherbal formulation. Indian J Traditional knowledge 7: 389-396. 